\title{
Clinical correlates of chronic cerebrospinal venous insufficiency in multiple sclerosis
}

\author{
Bianca Weinstock-Guttman ${ }^{1,2,5^{*}}$, Murali Ramanathan ${ }^{1,3}$, Karen Marr $^{4}$, David Hojnacki ${ }^{1}$, Ralph HB Benedict ${ }^{1}$, \\ Charity Morgan ${ }^{2}$, Eluen Ann Yeh', Ellen Carl ${ }^{3}$, Cheryl Kennedy ${ }^{4}$, Justine Reuther ${ }^{4}$, Christina Brooks ${ }^{4}$, \\ Kristin Hunt ${ }^{4}$, Makki Elfadil ${ }^{4}$, Michelle Andrews ${ }^{4}$ and Robert Zivadinov ${ }^{1,4}$
}

\begin{abstract}
Background: Chronic cerebrospinal venous insufficiency (CCSVI) is a vascular condition characterized by anomalies of the primary veins outside the skull that has been reported to be associated with MS. In the blinded Combined Transcranial (TCD) and Extracranial Venous Doppler Evaluation (CTEVD) study, we found that prevalence of CCSVI was significantly higher in multiple sclerosis (MS) vs. healthy controls (HC) $(56.1 \%$ vs. $22.7 \%, p<0.001)$.

The objective was to evaluate the clinical correlates of venous anomalies indicative of CCSVI in patients with MS.

Methods: The original study enrolled 499 subjects; 163 HC, 289 MS, 21 CIS and 26 subjects with other neurological disorders who underwent a clinical examination and a combined Doppler and TCD scan of the head and neck. This analysis was restricted to adult subjects with MS (RR-MS: $n=181$, SP-MS: $n=80$ and PP-MS: $n=12$ ). Disability status was evaluated by using the Kurtzke Expanded Disability Status Scale (EDSS) and MS severity scale (MSSS).

Results: Disability was not associated with the presence ( $\geq 2$ venous hemodynamic criteria) or the severity of CCSVI, as measured with venous hemodynamic insufficiency severity score (VHISS). However, the severity of CCSVI was associated with the increased brainstem functional EDSS sub-score $(p=0.002)$. In logistic regression analysis, progressive MS (SP-MS or PP-MS) vs. non-progressive status (including RR-MS) was associated with CCSVI diagnosis $(p=0.004, \mathrm{OR}=2.34, \mathrm{Cl}=1.3-4.2)$.
\end{abstract}

Conclusions: The presence and severity of CCVSI in multiple sclerosis correlate with disease status but has no or very limited association with clinical disability.

Search terms: Multiple sclerosis, Disease progression, Disability, Echo-color Doppler, Venous anomalies, CCSVI

\section{Background}

Multiple sclerosis (MS) is considered a chronic disease of the brain and spinal cord characterized by inflammatory-demyelinating and neurodegenerative features that coexist from early stages [1,2]. The heterogeneous clinical presentation and dissimilar long-term outcomes support a complex MS pathobiology. The recent findings of Zamboni et al. [3-5], who identified a strong association between MS and a condition defined as chronic cerebrospinal venous insufficiency (CCSVI) provide a different viewpoint on the possible cause and progression of MS. However, the relationship of

\footnotetext{
* Correspondence: BWeinstock-Guttman@Kaleidahealth.org

${ }^{1}$ Department of Neurology, State University of New York, Buffalo, NY, USA

${ }^{2}$ Department of Biostatistics, University of Alabama, Birmingham, AL, USA

Full list of author information is available at the end of the article
}

CCSVI to MS pathogenesis is unproven, and sensitivity/ specificity findings have ranged from perfect [3] to intermediate [6-13] to null association [14-19]. These contradictory research findings have fueled a polarizing controversy between practitioners and MS patients [2023]. Therefore a critical objective independent validation of the existence of CCSVI in MS patients and certainly establishing whether CCSVI is a cause, consequence or mere association with MS becomes imperative.

CCSVI has been described as a vascular condition characterized by anomalies of the main extra-cranial cerebrospinal (CS) venous routes that interfere with normal CS venous outflow. These anomalies have been reported to affect the internal jugular veins (IJV), the vertebral veins (VV) and the azygous vein (AZY), and can be detected using a noninvasive combined venous

\section{Biomed Central}


echo-color ultrasound and transcranial (ECD/TCD) Doppler and confirmed by selective venography [3-5]. It has been hypothesized that CS venous anomalies may cause alterations to blood flow that eventually result in iron deposition, decreased brain parenchyma metabolism, degeneration of neurons and characteristic brain injury patterns found in MS [24,25].

In an independent study [9] using identical EC/TCD method as described by Zamboni, our group showed recently a significant association between MS and presence of CCSVI compared to a healthy control group $(p<0.001)$. The goal of the present additional analysis within the initial large heterogeneous MS cohort was to determine the associations of venous hemodynamic findings according to the Zamboni CCSVI criteria with clinical disease severity and classification of disease status as relapsing or progressive.

\section{Methods}

\section{Study population}

Ethics statement

The study was approved by the University at Buffalo Human Subjects Institutional Review Board (HSIRB \#NEU2490109A). Written informed consent was obtained from all participants.

\section{Study design}

This cross-sectional study aims to evaluate the clinical correlates of a large cohort of MS patients in relation to the CCSVI criteria [3]. The study represents a post-hoc analysis of the original Combined Transcranial and Extracranial Venous ECD Evaluation (CTEVD) study which was designed to assess the prevalence of CCSVI in a large cohort of patients with MS, healthy controls and controls with other neurological diseases (OND) using specific ECD criteria [3,9]. This CTEVD phase 1 study enrolled $289 \mathrm{MS}$ and 21 clinically isolated syndrome (CIS) patients [9]. The participants received a clinical examination (BWG and $\mathrm{DH}$, that were blinded to the Doppler findings) and an ECD scan of the head and neck (performed by a technician blinded to the subjects' diagnosis) [9]. The clinical evaluation included neurological assessment using the Kurtzke Expended Disability Status Score (EDSS) based on its separate functional scores and gait endurance [26]. The EDSS was further converted to the MS Severity Scores (MSSS) scale that was designed as a comparative MS population disability assessment tool, to allow comparisons of relative disease severity at all EDSS levels for a given disease duration, using a single clinical assessment at a single point in time [27]. The ECD outcome measure of interest included the presence of CCSVI, as previously defined [3]. Additional ECD outcomes included the individual Doppler Criteria $1-5$ and CCSVI severity, as measured by venous hemodynamic insufficiency severity score (VHISS) [3].

\section{Echo-color doppler data analysis}

Combined transcranial and extracranial ECD allows for non-invasive assessment of venous hemodynamic (VH) parameters indicative of CCSVI $[3,4]$. Cerebral venous return was examined by using the echo-color Doppler (ECD Esaote-Biosound My Lab 25) equipped with 2.5 and 7.5-10 Mhz transducers (Genoa, Italy), with the subject positioned on a tilt bed at $90^{\circ}$ and $0^{\circ}[3,4]$.

The specific details of subject length of exam, contraindications and limitations, subject assessment, examination guidelines, annotation documentation, specific Doppler parameters, criteria definitions, description of probes, positioning of the subject, hydration status, techniques used, fulfilment of $\mathrm{VH}$ criteria and pathology definitions are provided elsewhere [9].

Each subject was assigned a total criteria VH score that was calculated by counting the number of criteria that the subject fulfilled. A subject was considered CCSVI-positive if $\geq 2 \mathrm{VH}$ criteria were fulfilled. Subjects who were not assessed for some $\mathrm{VH}$ criterion, due to technical difficulty, were assumed not to have fulfilled that criterion.

The five $\mathrm{VH}$ criteria evaluated are: Criterion 1) reflux in the IJVs and/or in the VVs assessed in both sitting and supine postures, Criterion 2) reflux in the deep cerebral veins (DCVs), Criterion 3) B-mode detection of stenoses in the IJVs in the form of annuli, webs, septa, or malformed valves, Criterion 4) absence of ECD signal in the IJV and/or in the VVs, even after forced deep breaths, and Criterion 5) presence of a negative difference in the cross sectional area (CSA) of the IJV. The severity of CCSVI was measured by VHISS, as previously reported [3]. VHISS is based on the sum of pathologic parameters measured for each of the 5 criteria examined. VHISS is ranging from 0 to 16.

\section{Data analysis}

SPSS (SPSS Inc., Chicago, IL, version 15.0) statistical program was used for all statistical analyses. The subjects with relapsing-remitting (RR) MS were categorized as non-progressive MS whereas subjects with relapsing and relapse-free forms of secondary progressive (SP) and primary-progressive (PP) MS were categorized as progressive MS.

One-way ANOVA followed by post-hoc independent sample $t$-tests were used to test for differences in means of continuous demographic variables such as age, age of onset, and disease duration. The $X^{2}$ test was used for analysis of count variables for categorical data and the Fisher exact test was used where appropriate. 
Logistic regression with the non-progressive vs. progressive MS status as the dependent variable, age as a covariate and gender as a factor was also used to assess the role of CCSVI. Analyses were conducted with main effects models containing CCSVI.

The EDSS values were dichotomized into EDSS $\geq 4$ and EDSS $<4.0$. The EDSS categories were analyzed as the dependent variable in logistic regression with sex and age and either presence of CCSVI or VHISS as predictors. The MSSS was analyzed as the dependent variable with linear regression with sex and age and either presence of CCSVI or VHISS as predictors. VHISS and number of venous hemodynamic criteria fulfilled were analyzed as dependent variable in Poisson regression with sex as a factor and age as a covariate. The EDSS sub-scores were analyzed with ordinal regression with age as a covariate, sex as a factor and either presence of CCSVI or VHISS as predictors.

In order to avoid too many spurious findings due to multiple comparisons, we do not report anything as statistically significant unless the nominal $p$-value was $\leq 0.01$ by using two-tailed tests. A trend was assumed with Type 1 error level of 0.1 .

\section{Results}

\section{Demographic and clinical characteristics}

The present analysis was restricted to adult subjects with RR-MS $(n=181)$, SP-MS $(n=80)$ and PP-MS $(n=12)$ disease courses. Healthy controls $(n=163)$, subjects with CIS $(n=21)$, pediatric-onset MS $(n=10)$, NMO $(n=6)$ and OND $(n=23)$ were excluded from the original cohort including 499 subjects [9].

Relapsing $(n=19)$ and non-relapsing SP-MS $(n=61)$ were grouped in to a single SP-MS category and similarly relapsing $(n=1)$ and non-relapsing primary $(n=11)$ progressive MS were grouped together in the PP-MS group. Table 1 shows demographic and clinical characteristics of the enrolled MS groups.

No age, sex or disease duration differences were found in the RR-MS and SP-MS in subjects either with or without the presence of CCSVI (all $p>0.05$ in $t$-tests for age and disease duration and $x^{2}$ test for sex differences, data not shown). The PP-MS patients with CCSVI were older $(59.3 \pm 6.3$ years $)$ than those without CCSVI $(49.8 \pm 6.8$ years $)$ but the sex distribution and disease duration were similar.

Of the 273 MS, 238/268 (88.8\%) were on diseasemodifying therapies; data were unavailable for 5 patients (Table 1). No differences in age at disease onset, disease diagnosis or number of relapses during the preceding year were seen between the CCSVI positive or negative groups (data not shown).

\section{Disease state and CCSVI}

Table 2 shows the prevalence of CCSVI classifications for MS patients by subtype of MS. The highest prevalence was seen in SP-MS, followed by PP-MS and RRMS. In logistic regression correcting for age and sex, progressive MS (SP-MS or PP-MS) vs. non-progressive status (RR-MS) was associated with CCSVI diagnosis $(p=0.004, \mathrm{OR}=2.34, \mathrm{CI}=1.3-4.2)$.

As expected, VHISS was significantly higher for MS patients diagnosed with CCSVI (Mean \pm SD: $4.20 \pm 1.4$ ) than for subjects without CCSVI (Mean \pm SD: $1.28 \pm 1.0 ; p<.001$ from a Mann-Whitney test). Table 2 gives the distribution of VHISS for the MS disease groups. The MS disease groups $(p<0.001$, Kruskall-Wallis) significantly differed in VHISS.

\section{Disability status and CCSVI diagnosis}

The three MS subtypes significantly differed on total EDSS score $(p<0.001$, Kruskall-Wallis test) and all EDSS subscores $(p<0.001$ for each, Kruskall-Wallis test). The probability of EDSS $\geq 4$ was not associated with the presence of CCSVI $(p=0.42)$ in logistic regression correcting for age and sex. In ordinal regression correcting for sex and age, the presence of CCSVI diagnosis exhibited a trend with the EDSS brain stem sub-score $(p=0.074)$.

Table 1 gives the distribution of MSSS for the MS disease groups. No evidence for differences in mean MSSS \pm SD for subjects diagnosed with CCSVI $(4.17 \pm 2.6)$ compared to subjects without CCSVI

Table 1 Demographic and clinical characteristics of the enrolled disease groups

\begin{tabular}{|c|c|c|c|c|c|}
\hline Demographic variables & $\begin{array}{l}\text { All MS } \\
n=273\end{array}$ & $\begin{array}{l}\text { RR-MS } \\
n=181\end{array}$ & $\begin{array}{l}\text { SP-MS } \\
n=80\end{array}$ & $\begin{array}{l}\text { PP-MS } \\
n=12\end{array}$ & $p$-value \\
\hline Females: Males (\% Female) & 208: 65 (76.2\%) & 138: 43 (76.2\%) & 63: $17(78.8 \%)$ & $7: 5(58.3 \%)$ & 0.34 \\
\hline Age, years & $47.7 \pm 10.7$ & $44.7 \pm 10.0$ & $53.3 \pm 9.7$ & $54.6 \pm 8.0$ & $<0.001$ \\
\hline Disease duration*, years & $15.2 \pm 10.5$ & $12.1 \pm 8.3$ & $21.9 \pm 12.0$ & $15.8 \pm 9.4$ & $<0.001$ \\
\hline Median EDSS* (IQR) & $3.0(4.0)$ & $2.0(1.5)$ & $6.0(1.5)$ & $5.75(3.13)$ & $<0.001$ \\
\hline MSSS & $4.05 \pm 2.51$ & $3.08 \pm 2.12$ & $5.87 \pm 2.11$ & $6.06 \pm 2.71$ & $<0.001$ \\
\hline Treatment duration, years & $3.8 \pm 3.7$ & $3.4 \pm 3.5$ & $4.8 \pm 4.1$ & $3.4 \pm 3.0$ & 0.03 \\
\hline
\end{tabular}

* From symptoms.

Female to male ratio: $x^{2}$ test; Age, disease duration, MSSS, treatment duration: one-way ANOVA; EDSS: Kruskal-Wallis test.

MS multiple sclerosis; $P P$ primary progressive, $R R$ relapsing-remitting; SP econdary progressive; IQR inter-quartile range; EDSS Expanded Disability Status Scale. 
Table 2 CCSVI classification by MS subtype

\begin{tabular}{|c|c|c|c|c|c|}
\hline CCSVI Variables & $\begin{array}{l}\text { All MS } \\
n=273\end{array}$ & $\begin{array}{l}\text { RR-MS } \\
n=181\end{array}$ & $\begin{array}{l}\text { SP-MS } \\
n=80\end{array}$ & $\begin{array}{l}\text { PP-MS } \\
n=12\end{array}$ & $p$-value \\
\hline CCSVI diagnosis & $153(56.0 \%)$ & $89 / 181(49.2 \%)$ & $58 / 80(72.5 \%)$ & $6 / 12(50.0 \%)$ & 0.002 \\
\hline Criterion 1 & $127(46.5 \%)$ & $76(42.0 \%)$ & $45(56.2 \%)$ & $6(50.0 \%)$ & 0.10 \\
\hline Criterion 2 & $98(35.9 \%)$ & $53(29.3 \%)$ & $40(50.0 \%)$ & $5(41.7 \%)$ & 0.005 \\
\hline Criterion 3 & $173(63.4 \%)$ & $115(63.5 \%)$ & $52(65.0 \%)$ & $6(50.0 \%)$ & 0.60 \\
\hline Criterion 4 & $28(10.3 \%)$ & $12(6.6 \%)$ & $15(18.8 \%)$ & $1(8.3 \%)$ & 0.012 \\
\hline Criterion 5 & $31(11.4 \%)$ & $12(6.6 \%)$ & $19(23.8 \%)$ & $0(0 \%)$ & $<0.001$ \\
\hline Number of abnormal criteria & $1.7 \pm 1.1$ & $1.5 \pm 1.1$ & $2.2 \pm 1.1$ & $1.5 \pm 1.0$ & $<0.001$ \\
\hline VHISS & $2.9 \pm 1.9$ & $2.6 \pm 1.8$ & $3.7 \pm 2.0$ & $2.5 \pm 1.6$ & $<0.001$ \\
\hline
\end{tabular}

MS multiple sclerosis; $P P$ primary progressive, $P R$ progressive relapsing; $R R$ relapsing-remitting; $S P$ secondary progressive; VHISS venous hemodynamic insufficiency score.

(3.88 $\pm 2.3, p=0.60$ from a Mann-Whitney test) were found. Similarly linear regression analyses (correcting for sex and age) did not show association with CCSVI $(p=0.49)$.

\section{Disability status and CCSVI severity}

The VHISS exhibited a trend with increasing age (Slope B \pm SE: $0.007 \pm 0.0033$, Wald $\chi^{2}=4.54, p=0.033$ ) in Poisson regression with sex as a factor and age as a covariate. However there was no evidence for associations with EDSS.

VHISS was associated with the brainstem EDSS subscore $(p=0.002)$ and trends were found for the pyramidal $(p=0.026)$ and cerebellar $(p=0.051)$ sub-scores. The probability of EDSS $\geq 4$ was not associated with the severity of CCSVI as assessed by VHISS $(p=0.056)$ in logistic regression correcting for age and sex.

\section{Discussion and conclusion}

The present study, a post hoc analysis of the original CTEVD study, which aimed primarily to determine the prevalence of CCSVI in MS patients, provides supplementary informative data to better understand the relation between MS and CCSVI criteria with a specific emphasis on disease and disability status. The original CTEVD study showed an increased prevalence of CCSVI in MS but substantially lower than the originally reported than the $100 \%$ sensitivity and $100 \%$ specificity reported by Zamboni et al. [3] as only $56.1 \%$ of MS patients and $38.1 \%$ of CIS patients presented with abnormal venous ECD/TCD [9].

Bastianello et al. in a recent multicenter study evaluating 710 patients and using the Zamboni criteria [3] showed a significant association between presence of CCSVI (identified in 86\%) and progressive disease [28]. The positive CCSVI patients had also higher disability and a later disease onset as compared to the MS patients without CCSVI. We can speculate that the presence of CCSVI related to certain structural abnormalities as incompetent valves or abnormal intra-luminal structures as septums, flaps or webs can worsen with aging and/or disease progression [29]. In fact, another study showed that CCSVI was diagnosed in $50 \%$ of MS patients but was seen also in $36 \%$ of the healthy controls [30]. No correlation between presence of CCSVI and EDSS or progressive disease was found, although this can be related to the small number of progressive patients (15/ 84) enrolled in this study [30]. Another smaller scale study found no association between disease status and CCSVI [16].

Although our large prevalence study showed higher frequency of CSSVI in progressive MS compared to relapsing MS, we did not obtain evidence for significant associations with disability. The divergence of the prevalence and disability data adds to the known difficulties of understanding and measuring the effects of the two key pathological mechanisms in MS - inflammatory vs. neurodegeneration - by introducing the presence of a $\mathrm{VH}$ dysfunction as a new variable. Hemodynamic venous dysfunction may result in secondary tissue hypoperfusion that may be associated with a continuous neurodegenerative process. Although VH dysfunction may be less related to the inflammatory aspects of the disease, we speculate that abnormal $\mathrm{VH}$ may progressively deteriorate in the context of the inflammatory milieu and activation of the vascular endothelium that occurs in MS [31]. Our previous findings in a OND group showing a high prevalence of CCSVI in the majority of patients diagnosed with inflammatory diseases (i.e., anti-phospholipid antibody syndrome, thyroiditis) [9]. CCSVI findings, if present at all, may reflect: i) benign anatomical variants ii) slower venous outflow due to global cerebral hypoperfusion or iii) alterations to venous walls secondary to a chronic inflammation milieu.

In conclusion, our results indicate that features of CCSVI have no relationship to disability in MS. Further prospective studies including multimodal analysis (i.e., MRI/MRV, ECD/TCD) may help in providing more light into the role of CCSVI or of VH dysfunction in MS. 


\section{Competing interests}

Dr. Weinstock-Guttman received compensation for speaking from Teva Neuroscience, Biogen Idec, EMD Serono and Novartis. She also received financial support for research activities from National Institute of Health, National Multiple Sclerosis Society, National Science Foundation, EMD Serono, Biogen Idec, Teva Neuroscience, Novartis, Cyberonics and the Jog for the Jake Foundation.

Murali Ramanathan received research funding or consulting fees from EMD Serono, Biogen Idec, Allergan, Netezza, Pfizer, Novartis, the National Multiple Sclerosis Society, the Department of Defense, Jog for the Jake Foundation, the National Institutes of Health and National Science Foundation. He received compensation for serving as an Editor from the American Association of Pharmaceutical Scientists. These are unrelated to the research presented in this report.

Ralph Benedict has received compensation for serving on Advisory Panels for Biogen, EMD Serono, Merck, Novartis, and Bayer. He has received CME funding from Bayer, Merck, and Biogen and grant support from NMSS, NIH, Biogen, and Shire.

Dr. Zivadinov has received speaker honoraria and consultant fees from Teva Neurosciences, Biogen Idec, Questcor, Genzyme and EMD Serono; and received research support from the National Multiple Sclerosis Society, the Biogen Idec, Teva Neuroscience, Teva Pharmaceuticals, Genzyme, Questcor, Bracco and Greatbatch.

This study was funded by internal resources of the Buffalo Neuroimaging Analysis Center and Baird MS Center, the Jacobs Neurological Institute, University of Buffalo. In addition, we received support from the Direct MS Foundation, the Jacquemin Family Foundation and from smaller donors. The results from the CTEVD study led to the organization of the IRB approved, unblinded, open-label descriptive study into CCSVI that included patients with either possible or definite MS. This fee-for-service registry study was designed to enhance utilization of data on venous anomalies obtained on individuals who sought information on CCSVI status. This study is no longer active.

\section{Authors' contributions}

BW-G contributed to study design, oversaw all clinical aspects of the project including clinical data acquisition, data analysis and interpretation and manuscript preparation. MR contributed to study design, data analysis and interpretation and manuscript preparation. RZ contributed to study design, MRI data acquisition, data interpretation and manuscript preparation. KM contributed to Doppler data acquisition. DH contributed to clinical data acquisition and interpretation. RHBB contributed to study design and interpretation of findings. CM contributed to data analysis. EAY contributed to patient recruitment and clinical data acquisition. EC contributed to data acquisition and analysis. CK, JR, CB, KH and MA contributed to patient recruitment and study coordination. ME contributed to Doppler data acquisition. All authors read and approved the final manuscript.

\section{Author details}

'Department of Neurology, State University of New York, Buffalo, NY, USA. ${ }^{2}$ Department of Biostatistics, University of Alabama, Birmingham, AL, USA. ${ }^{3}$ Department of Pharmaceutical Sciences, State University of New York, Buffalo, NY, USA. ${ }^{4}$ Buffalo Neuroimaging Analysis Center, Department of Neurology, State University of New York, Buffalo, NY, USA. ${ }^{5}$ Department of Neurology, State University of New York, Buffalo, E2 Neurology, Buffalo General Hospital, 100 High Street, Buffalo, NY 14203, USA.

Received: 3 August 2011 Accepted: 3 April 2012

Published: 15 May 2012

\section{References}

1. Frohman EM, Racke MK, Raine CS: Multiple sclerosis-the plaque and its pathogenesis. N Engl J Med 2006, 354(9):942-955.

2. Frischer JM, Bramow S, Dal-Bianco A, Lucchinetti CF, Rauschka H, Schmidbauer M, Laursen H, Sorensen PS, Lassmann H: The relation between inflammation and neurodegeneration in multiple sclerosis brains. Brain 2009, 132(Pt 5):1175-1189.

3. Zamboni P, Galeotti R, Menegatti E, Malagoni AM, Tacconi G, Dall'ara S, Bartolomei I, Salvi F: Chronic cerebrospinal venous insufficiency in patients with multiple sclerosis. J Neurol Neurosurg Psychiatry 2009, 80 (4):392-399.
4. Zamboni P, Menegatti E, Galeotti R, Malagoni AM, Tacconi G, Dall'Ara S, Bartolomei I, Salvi F: The value of cerebral Doppler venous haemodynamics in the assessment of multiple sclerosis. J Neurol Sci 2009, 282(1-2):21-27.

5. Zamboni P, Menegatti E, Weinstock-Guttman B, Schirda C, Cox JL, Malagoni AM, Hojanacki D, Kennedy C, Carl E, Dwyer MG, et al: The severity of chronic cerebrospinal venous insufficiency in patients with multiple sclerosis is related to altered cerebrospinal fluid dynamics. Funct Neurol 2009, 24(3):133-138.

6. Simka M, Kostecki J, Zaniewski M, Majewski E, Hartel M: Extracranial Doppler sonographic criteria of chronic cerebrospinal venous insufficiency in the patients with multiple sclerosis. Int Angiol 2010, 29(2):109-114

7. Al-Omari MH, Rousan LA: Internal jugular vein morphology and hemodynamics in patients with multiple sclerosis. Int Angiol 2010, 29(2):115-120.

8. Yamout B, Herlopian A, Issa Z, Habib RH, Fawaz A, Salame J, Wadih A, Awdeh $H$, Muallem N, Raad R, et al: Extracranial venous stenosis is an unlikely cause of multiple sclerosis. Mult Scler 2010, 16(11):1341-1348.

9. Zivadinov R, Marr K, Cutter G, Ramanathan M, Benedict RH, Kennedy C, Elfadil M, Yeh AE, Reuther J, Brooks C, et al: Prevalence, sensitivity, and specificity of chronic cerebrospinal venous insufficiency in MS. Neurology 2011, 77(2):138-144.

10. Monti L, Menci E, Ulivelli M, Cerase A, Bartalini S, Piu P, Marotti N, Leonini S, Galluzzi P, Romano DG, et al: Quantitative ColourDopplerSonography evaluation of cerebral venous outflow: a comparative study between patients with multiple sclerosis and controls. PLoS One 2011, 6(9):e25012.

11. Zaharchuk G, Fischbein NJ, Rosenberg J, Herfkens RJ, Dake MD: Comparison of MR and contrast venography of the cervical venous system in multiple sclerosis. AJNR Am J Neuroradiol 2011, 32(8):1482-1489.

12. Radak D, Tanaskovic S, Marinkovic S, Antonic Z, Kolar J: Internal jugular vein duplication: a further truncular malformation in a patient with multiple sclerosis. Phlebology 2011 Oct 28, [Epub ahead of print]

13. Radak D, Kolar J, Tanaskovic S, Sagic D, Antonic Z, Mitrasinovic A, Babic S, Nenezic D, llijevski N: Morphological and haemodynamic abnormalities in the jugular veins of patients with multiple sclerosis. Phlebology 2011 Sep 8, [Epub ahead of print]

14. Doepp F, Paul F, Valdueza JM, Schmierer K, Schreiber SJ: No cerebrocervical venous congestion in patients with multiple sclerosis. Ann Neurol 2010, 68(2):173-183.

15. Mayer CA, Pfeilschifter W, Lorenz MW, Nedelmann M, Bechmann I, Steinmetz H, Ziemann U: The perfect crime? CCSVI not leaving a trace in MS. J Neurol Neurosurg Psychiatry 2011, 82(4):436-440.

16. Baracchini C, Perini P, Causin F, Calabrese M, Rinaldi F, Gallo P: Progressive multiple sclerosis is not associated with chronic cerebrospinal venous insufficiency. Neurology 2011, 77(9):844-850.

17. Tsivgoulis G, Mantatzis M, Bogiatzi C, Vadikolias K, Voumvourakis $\mathrm{K}$, Prassopoulos P, Piperidou C, Heliopoulos I: Extracranial venous hemodynamics in multiple sclerosis: a case-control study. Neurology 2011, 77(13):1241-1245.

18. Auriel E, Karni A, Bornstein NM, Nissel T, Gadoth A, Hallevi H: Extra-cranial venous flow in patients with multiple sclerosis. J Neurol Sci 2011, 309(1-2):102-104.

19. Marder E, Gupta P, Greenberg BM, Frohman EM, Awad AM, Bagert B, Stuve $O$ : No cerebral or cervical venous insufficiency in US veterans with multiple sclerosis. Arch Neurol 2011, 12:1521-1525.

20. Zamboni P, Galeotti R, Menegatti E, Malagoni AM, Gianesini S, Bartolomei I, Mascoli F, Salvi F: A prospective open-label study of endovascular treatment of chronic cerebrospinal venous insufficiency. J Vasc Surg 2009, 50(6):1348-1358. e1341-e1343.

21. Reekers JA, Lee MJ, Belli AM, Barkhof F: Cardiovascular and interventional radiological society of Europe commentary on the treatment of chronic cerebrospinal venous insufficiency. Cardiovasc Intervent Radiol 2011, 34(1):1-2.

22. Krogias C, Schroder A, Wiendl H, Hohlfeld R, Gold R: "Chronic cerebrospinal venous insufficiency" and multiple sclerosis: critical analysis and first observation in an unselected cohort of MS patients. Nervenarzt 2010, 81(6):740-746. 
23. Baracchini C, Perini P, Calabrese M, Causin F, Rinaldi F, Gallo P: No evidence of chronic cerebrospinal venous insufficiency at multiple sclerosis onset. Ann Neurol 2011, 69(1):90-99.

24. Singh AV, Zamboni P: Anomalous venous blood flow and iron deposition in multiple sclerosis. J Cereb Blood Flow Metab 2009, 29(12):1867-1878.

25. Zamboni P: The big idea: iron-dependent inflammation in venous disease and proposed parallels in multiple sclerosis. J R Soc Med 2006, 99 (11):589-593.

26. Kurtzke JF: Rating neurologic impairment in multiple sclerosis: an expanded disability status scale (EDSS). Neurology 1983, 33(11):1444-1452.

27. Roxburgh RH, Seaman SR, Masterman T, Hensiek AE, Sawcer SJ, Vukusic S, Achiti I, Confavreux C, Coustans M, le Page E, et al: Multiple sclerosis severity score: using disability and disease duration to rate disease severity. Neurology 2005, 64(7):1144-1151.

28. Bastianello S, Romani A, Viselner G, Colli Tibaldi E, Giugni E, Altieri M, Cecconi P, Mendozzi L, Farina M, Mariani D, et al: Chronic cerebrospinal venous insufficiency in multiple sclerosis: clinical correlates from a multicentre study. BMC Neurology 2011, 11:132.

29. Dolic K, Marr K, Valnarov V, Dwyer MG, Carl E, Karmon Y: Intra- and extraluminal structural and functional venous anomalies in multiple sclerosis, as evidenced by two non-invasive imaging techniques. Am J Neuroradiol 2011, press.

30. Centonze D, Floris R, Stefanini M, Rossi S, Fabiano S, Castelli M, Marziali S, Spinelli A, Motta C, Garaci FG, et al: Proposed chronic cerebrospinal venous insufficiency criteria do not predict multiple sclerosis risk or severity. Ann Neurol 2011, 70(1):52-59.

31. Minagar A, Carpenter A, Alexander JS: The destructive alliance: interactions of leukocytes, cerebral endothelial cells, and the immune cascade in pathogenesis of multiple sclerosis. Int Rev Neurobiol 2007, 79:1-11.

doi:10.1186/1471-2377-12-26

Cite this article as: Weinstock-Guttman et al: Clinical correlates of chronic cerebrospinal venous insufficiency in multiple sclerosis. BMC Neurology 2012 12:26.

\section{Submit your next manuscript to BioMed Central and take full advantage of:}

- Convenient online submission

- Thorough peer review

- No space constraints or color figure charges

- Immediate publication on acceptance

- Inclusion in PubMed, CAS, Scopus and Google Scholar

- Research which is freely available for redistribution 